\title{
Effectiveness of Estrogen Replacement in Restoration of Cognitive Function after Long-Term Estrogen Withdrawal in Aging Rats
}

\author{
Alicja L. Markowska and Alena V. Savonenko \\ Neuromnemonics Laboratory, Department of Psychology, Johns Hopkins University, Baltimore, Maryland 21218
}

Recent studies suggest that some aspects of learning and memory may be altered by a midlife loss of estrogen, indicating a potential causal relationship between the deficiency of ovarian hormones and cognitive aging. In this study, the effects of estrogen withdrawal and replacement were tested in middleaged Fischer-344 rats using different memory tasks. Estrogen withdrawal accelerated the rate of cognitive aging. A deficit first occurred 4 months after ovariectomy in working memory, which was tested in a delayed-nonmatching-to-position task, and progressed from long-delay to short-delay trials. Reference memory, which was tested in a place discrimination task and a split-stem T-maze, was not affected by aging or ovariectomy. The efficacy of estrogen in ameliorating the cognitive deficit in old rats depended on the type of treatment (acute vs chronic) and whether the aging-related decline in a particular cognitive process was aggravated by estrogen withdrawal. Chronic es- trogen treatment (implants) was effective in improving working memory only when primed with repeated injections of estrogen, indicating that simulating the estrogen fluctuations of the estrous cycle may be more effective than the widely used mode of chronic pharmacological treatment. A challenge with scopolamine revealed that ovariectomy-induced cognitive deterioration coincided with a compromised cholinergic system. Importantly, the estrogen treatment that had restored effectively the cognitive abilities of old ovariectomized rats did not reduce their sensitivity to scopolamine. Taking into consideration that estrogen was highly effective against the amnestic action of scopolamine when tested in young-adult rats, these data emphasize that mechanisms of the protective effect of estrogen differ in young and old rats.

Key words: ovariectomy; cholinergic system; scopolamine; working memory; attention; body weight
Recent studies suggest that some aspects of learning and memory may be altered by the midlife loss of estrogen (Sherwin, 1988). These changes can be ameliorated by estrogen replacement therapy (Ditkoff et al., 1991; Sherwin, 1994; Kimura, 1995), indicating a potential causal relationship between the deficiency of ovarian hormones and cognitive aging. In previous studies conducted on animal models, estrogen replacement has been shown to improve the performance of ovariectomized rats in radial mazes (Daniel et al., 1997; Luine et al., 1998; Fader et al., 1999) and water mazes (O'Neal et al., 1996), in the operant alternation task (Dohanich et al., 1994; Fader et al., 1998), and in the active avoidance task (Singh et al., 1994). However, because only two groups of rats (ovariectomized and estrogen-replaced) generally have been used, it is impossible to determine whether the superiority of the estrogen-replaced rats was attributable to a cognition-enhancing effect of estrogen or to a deleterious effect of estrogen deprivation in the ovariectomized rats. If aging is considered as an intervening factor, the impact of these effects is likely to change significantly.

Our previous studies have indicated that there is no cognitive deficit immediately after ovariectomy, and that the deleterious effect on cognition is delayed for a longer period of time in young females than in older females (Markowska and Grinnell, 1999).

\footnotetext{
Received May 20, 2002; revised Oct. 3, 2002; accepted Oct. 3, 2002.

This study was supported by National Institute on Aging Grant AG15947 to A.L.M. We thank Y. Aguirre, J. L. Morton, K. Phelan, and D. Waters for their assistance with behavioral testing and J. L. Morton and L. Sims for editorial assistance.

Correspondence should be addressed to Alicja L. Markowska, National Institute on Aging, Gateway Building, Suite 2C-212, Bethesda, MD 20892. E-mail: markowsa@nia.nih.gov.

Copyright (C) 2002 Society for Neuroscience $0270-6474 / 02 / 2210985-11 \$ 15.00 / 0$
}

Therefore, aging should be considered as a factor that increases the susceptibility of cognitive function to the effects of estrogen withdrawal. Likewise, the effect of estrogen replacement varies depending on the age of females and the duration of treatment (Gibbs, 1998, 2000b; Gibbs and Aggarwal, 1998).

The first goal of this study was to assess the effects of estrogen withdrawal on the rate of cognitive aging in two groups of middle-aged female rats: (1) the control group that had intact ovaries and naturally decreasing estrogen levels during aging and (2) the ovariectomized rats that had undergone aging along with a surgically reduced level of estrogen. Middle age coincides with the onset of an age-related loss of ovarian hormones; therefore, middle-aged female rats may be an ideal model to test the effects of estrogen replacement on memory during aging (Markowska, 1999b).

When tested in young-adult rats, the duration and modes of estrogen treatment have been shown to be critical factors in determining the physiological (Gorzalka and Moe, 1994; Reilly et al., 1996), neuronal (Gibbs, 1998, 2000a), and cognitive effects (Luine et al., 1998) of treatment. These factors also may modulate the effectiveness of estrogen replacement on cognition during aging. Therefore, the second goal of this study was to characterize the effectiveness of estrogen replacement in middle-aged and old ovariectomized rats by using various durations and two modes of estrogen administration: acute injections and chronic implants. The acute mode of treatment was designed to simulate a naturally occurring fluctuation in the estrogen level during the estrus cycle (the injections were omitted every fourth day) and to reduce the probability of side effects by using a phasic, but not tonic, replacement. The chronic mode of treatment was used as a regimen that models more closely the pharmacological replacement of estro- 
gen. The assessment of these two treatments is particularly important in view of the recent findings from clinical trials in which daily administration of drugs containing a pharmacological dose of estrogen induced undesirable side effects in women (Grady et al., 2002; Hulley at al., 2002).

The third goal of this study was to evaluate the ability of estrogen to enhance cholinergic function during aging. A muscarinic antagonist, scopolamine (SCOP), was used before and after the estrogen treatment in the old females to estimate the efficacy of estrogen in counterbalancing the amnestic effects.

\section{MATERIALS AND METHODS}

\section{Subjects}

Thirteen-month-old Fischer-344 female rats $(n=30)$ were obtained from the colony at the National Institute of Aging. The rats (three rats per cage) were housed on a $12 \mathrm{hr}$ light/dark cycle. Food and water were provided ad libitum except for the periods of behavioral testing in the appetitively motivated tasks, in which water deprivation was necessary to create appropriate motivation for the task (for details, see DNMP task). To avoid the estrogen-like effect of phytoestrogens, which are found in many laboratory animal diets (Thigpen et al., 1999), the rats were fed a special diet without phytoestrogen content (5K96C; Purina Test Diet, Richmond, IN).

\section{Group treatment}

After a $5 \mathrm{~d}$ acclimation period, rats were divided randomly into two groups. One group $(n=20)$ received ovariectomy $(\mathrm{OVX})$, whereas the other group $(n=10)$ received sham surgery with skin and muscles cut but ovaries spared (Sham). After the recovery period ( $3 \mathrm{~d})$, each rat was handled for 5-10 min daily over $5 \mathrm{~d}$.

The rats were tested repeatedly during seven testing periods (periods 1-7), starting 1 month after ovariectomy and continuing for the next 9 months (Fig. 1). Behavioral testing included the delayed-nonmatchingto-position (DNMP) task, the spontaneous alternation task, place and visual discrimination, straight swim, and repeated acquisition in the water maze.

Six months after ovariectomy, during period 5, the OVX rats were divided randomly into two subgroups. The first half of OVX rats $\left(\mathrm{OVX}_{1}\right.$ group) and Sham rats were injected subcutaneously with $0.1 \mathrm{ml}$ of sesame oil, whereas the second half of OVX rats $\left(\mathrm{OVX}_{2}\right)$ received subcutaneous injections of estrogen $(10 \mu \mathrm{g}$ of $17 \beta$-estradiol dissolved in $0.1 \mathrm{ml}$ of sesame oil). Next, $5 \mathrm{~d}$ before period $6, \mathrm{OVX}_{2}$ rats were given estrogen implants. The $\mathrm{OVX}_{1}$ rats received estrogen implants $5 \mathrm{~d}$ before period 7. Sham rats received placebo implants. To control for neuroendocrine status, vaginal smears were obtained daily during $20 \mathrm{~d}$ in each of the seven testing periods. Body weight was recorded twice weekly during the course of the experiment.

\section{SCOP challenges}

During period 4, both Sham and OVX rats received intraperitoneal injections of saline $(0.9 \% \mathrm{NaCl}$ solution; Sigma, St. Louis, MO), SCOP $(0.05,0.1$, and $0.2 \mathrm{mg} / \mathrm{kg}$ in saline; Sigma), or methylscopolamine $(0.2$ $\mathrm{mg} / \mathrm{kg}$ in saline; Sigma) $20 \mathrm{~min}$ before behavioral testing. Each drug and dose was applied three times in a semirandom order with intertreatment intervals of 3-4 d.

During period 5, SCOP was administered to Sham, $\mathrm{OVX}_{1}$, and $\mathrm{OVX}_{2}$ rats twice; in the $\mathrm{OVX}_{2}$ group, $\mathrm{SCOP}$ was administered after the second and the fourth set of estrogen injections. Because at that time all rats were already 20 months of age, only the minimal dose of SCOP $(0.05$ $\mathrm{mg} / \mathrm{kg}$ ) was used.

\section{Ovariectomy}

The rats were anesthetized with an intraperitoneal injection of Nembutal (40 mg/kg; Abbott Labs, Abbott Park, IL). Fur on both sides of the body was shaved from a hip to the lowest rib. Bilateral ovariectomies were performed using an incision $1.5 \mathrm{~cm}$ inferior to the palpated rib cage. Ovaries and surrounding fat tissue were removed; the incision was closed by suturing the muscles and stapling the skin. Antibiotic gel was applied on the wound, and the animal was placed on paper towels in a temperature-controlled cage until awakening.

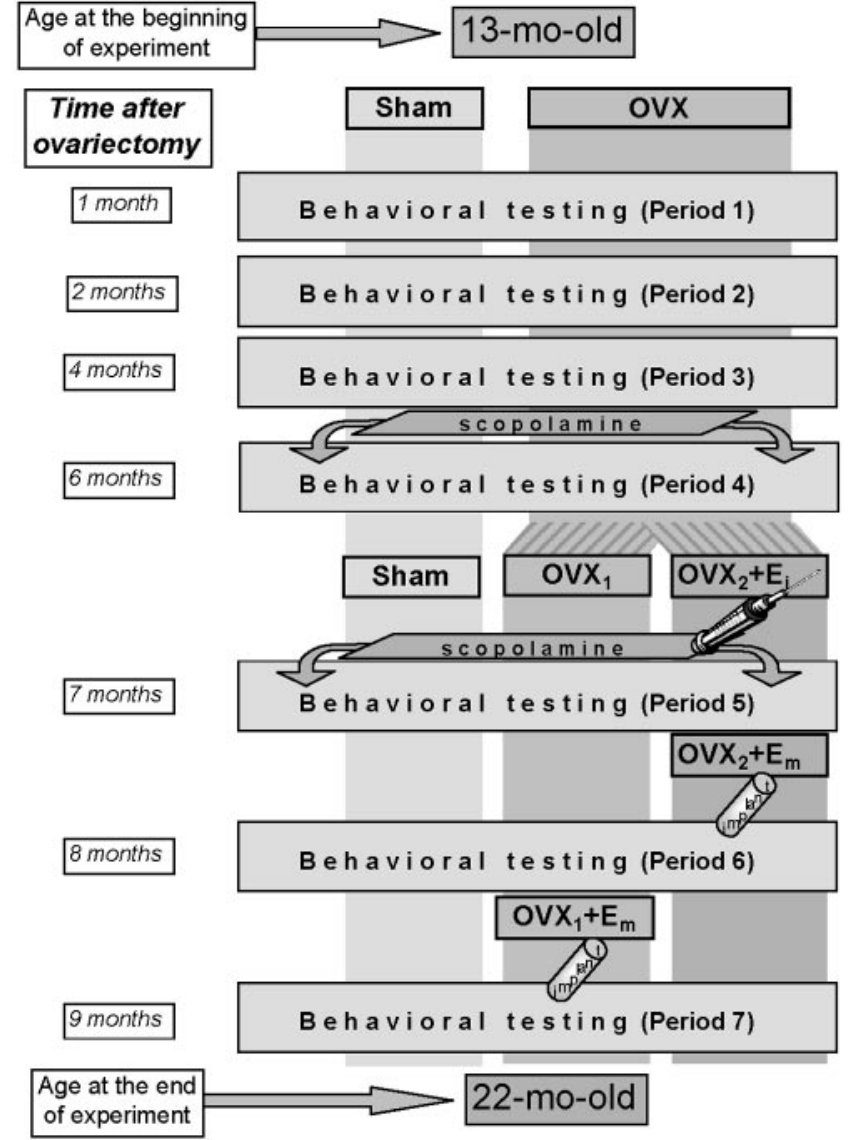

Figure 1. Design of the experiment and group treatment. Sham, Control rats with sham surgery in which ovaries were spared. $O V X$, Ovariectomized rats. $E$, Treatment with $17 \beta$-estradiol: $i$, acute, with injections; $m$, chronic, with implants. Injections of SCOP were applied during periods 4 and 5 .

\section{Vaginal cytology}

To monitor the stages of estrus cyclicity, cell samples were obtained by vaginal lavage, fixed, and stained in accordance with the Wright-Giemsa method (Hema 3, Fisher Scientific, Houston, TX). Next, the vaginal smears were investigated under the microscope and diagnosed for estrus stage (i.e., estrus, diestrus, or proestrus). Estrus stage was defined by the predominance of cornified cells in the vaginal lumen. The following period in which the cornified cells became less numerous and leukocytes and nucleated cells were present was defined as diestrus. Proestrus was defined as the time during which the vaginal smear was characterized by the predominance of nucleated epithelial cells (Feder, 1981). The duration of the estrus cycle was estimated as a number of days between consecutive estrus stages.

\section{Estrogen treatments}

The subcutaneous injections of estrogen contained $10 \mu \mathrm{g}$ of $17 \beta$-estradiol (Sigma) dissolved in $0.1 \mathrm{ml}$ of sesame oil (Sigma). The dose of estrogen chosen in this study was shown to be effective in inducing the structural synaptic changes in the hippocampus in young rats (Woolley and McEwen, 1994), and it partially restored levels of choline acetyltransferase mRNA in the medial septum in old rats (Gibbs and Aggarwal, 1998). Each rat from group $\mathrm{OVX}_{2}$ received multiple estrogen injections, scheduled as shown in Figure 2. 17 $\beta$-Estradiol injections for 3 consecutive days with no injection on the fourth day constitute one treatment set. This schedule of estrogen injections approximately models the natural drop in the level of estrogen every fourth day of the estrus cycle. Injections were given after behavioral training on days 1 and 2 and before the training on day 3 . Rats from the $\mathrm{OVX}_{2}$ group received four sets of estrogen injections with $12 \mathrm{~d}$ between set 2 and set 3 . During this period, SCOP was tested twice (see SCOP challenges). 


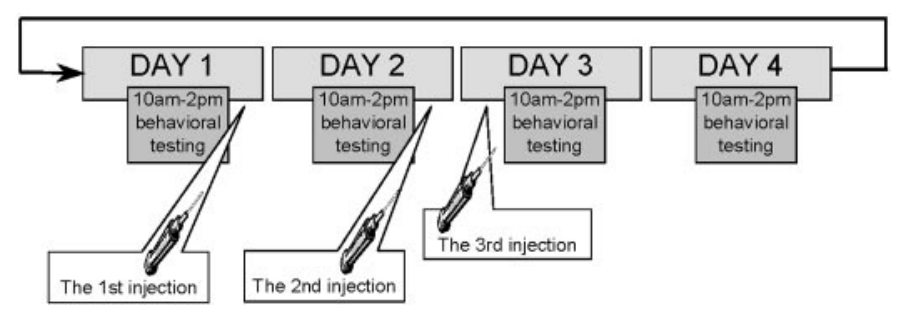

Figure 2. The schedule of $17 \beta$-estradiol injections. One set consisted of the three injections as shown in the figure. Ovariectomized rats (group $\mathrm{OVX}_{2}$ ) received four sets of injections.

Silastic tubing pellets (length, $5 \mathrm{~mm}$; inner diameter, $1.47 \mathrm{~mm}$ ), filled for $3 \mathrm{~mm}$ with $17 \beta$-estradiol, were placed in saline solution $(0.9 \% \mathrm{NaCl})$ at $37^{\circ} \mathrm{C}$ for $24 \mathrm{hr}$ before implantation. The pellet then was implanted under the skin of the neck when the rat was anesthetized with a gas anesthesia (a mixture of $\mathrm{O}_{2}, \mathrm{~N}_{2} \mathrm{O}_{2}$, and enflurane; Ohmeda, Liberty Corner, NJ). Behavioral testing started $5 \mathrm{~d}$ after implantation.

\section{Sampling estradiol concentration}

Once per month, the rats were anesthetized with gas anesthesia (see above), and a vein on the side of the tail was cut 5-6 cm up from the tip. Using peristaltic motion, $1 \mathrm{ml}$ of blood was collected into an Eppendorf tube containing $0.1 \mathrm{ml}$ of heparin. Plasma was collected by spinning the tubes for $30 \mathrm{~min}$ at $4^{\circ} \mathrm{C}$ and then stored at $-70^{\circ} \mathrm{C}$ until ready for ${ }^{125}$ I-radioimmunoassay for $17 \beta$-estradiol (DSL-4800 kit; Diagnostic Systems, Webster, TX).

\section{Behavioral testing \\ DNMP task}

Rats were tested in a split-stem T-maze that has been described previously (Hepler et al., 1985; Markowska et al., 1990). The 50-cm-long stem was divided into two halves by a partition. At the end of the partition proximal to the arms, a black cloth curtain was placed on both sides of the stem. A clear Plexiglas barrier placed behind one curtain blocked access to the arms on that side of the stem. The correct side of the stem was assigned randomly to rats but remained constant for each rat throughout the experiment. Each arm of the maze was $37 \mathrm{~cm}$ long and had a food cup $1 \mathrm{~cm}$ from the end. Chocolate milk was used as reinforcement for choosing the correct arm. To prevent possible orientation by the smell of the milk, several other cups filled with milk were placed beneath the stem and arms. Background masking noise was provided by radio speakers. The location of extra maze cues and speakers was held constant throughout the experiment.

The rats were deprived of water for $12 \mathrm{hr}$. Each daily session consisted of 11 trials. For the first, forced trial, one arm of the maze was blocked and the rat entered the opposite (baited) arm. After reinforcement, the rat was placed back into the home cage for an intertrial interval. During the next 10 trials, the rat was allowed to choose arms freely, and reinforcement was placed on the side of the maze that was not visited during the previous trial. After each trial, the maze was cleaned with a dilute alcohol solution and dried with a paper towel. During the first testing in DNMP (period 1), acquisition training was conducted with a minimal intertrial delay $(20-25 \mathrm{sec})$ and continued up to the criteria of $85 \%$ correct choices over 2 consecutive days. After the rat reached the criteria, longer intertrial delays were introduced $(1,5,15$, or $30 \mathrm{~min})$. Each delay was used for three sessions and scheduled in a semirandom order. The number of correct choices in the stem and arms and the maximum consecutive correct arm choices in each session were measured.

\section{Water maze tasks}

Water maze apparatus. A large metal pool $(180 \mathrm{~cm}$ in diameter $)$ filled with opaque water at $24 \pm 2^{\circ} \mathrm{C}$ and a $10 \mathrm{~cm}$ platform were used in place discrimination, repeated acquisition, and visual discrimination tasks. A straight plastic alley $(10 \times 70 \mathrm{~cm})$ was placed in the large pool and used during the straight swim test to estimate swimming ability. To prevent fatigue and changes in thermoregulation, the rat was removed from the pool and dried with a towel after each trial. The tracking system (HVS Image Analysis VP-200; HVS Image, Hampton, UK) acquired images from a camera mounted $1.4 \mathrm{~m}$ above the surface of the water. Eight $40 \mathrm{~W}$ bulbs, mounted in a parallel configuration $1.2 \mathrm{~m}$ above the surface of the water, provided lighting.

Straight swim test. This test, described in detail previously (Markowska et al., 1993), assessed the swimming ability of the rat in the straight alley and was conducted for two sessions of five trials each. The latency and swim speed to reach the platform at the end of the straight alley were recorded. The test was conducted $1 \mathrm{~d}$ before the place discrimination task.

Place discrimination task. This task has been described in detail previously (Markowska et al., 1993, 1998). Each rat received $1 \mathrm{~d}$ of training with three sessions of five platform trials and one probe trial each and a random order of start positions. During the platform trials, the platform was submerged but accessible to the rat, whereas during the probe trials, the platform was collapsed at the bottom of the tank for variable intervals $(10-40 \mathrm{sec})$ to test the subject's preference for the platform location. The collapsed platform was then returned to its raised position at the end of the probe trial to maintain the same response-reinforcement contingency as in the platform trials (Markowska et al., 1993). Latency [the time to reach the platform from the start location (in seconds)], distance [the path from the start location to the platform (in centimeters)], and swim speed [the average speed during a trial (in centimeters per second)] were measured during the platform trials. A lower score in the platform measures (except for swim speed) indicates better performance. In the probe trials, a percentage of time spent in the correct quadrant was assessed. Higher scores in this index reflected better performance. Location of the platform and a set of spatial cues around the tank were changed between the testing periods. The test was conducted during periods $1-3,6$, and 7 (see Fig. 1).

Repeated acquisition task. The training started on the day after the place discrimination task and consisted of $1 \mathrm{~d}$ of training with two sessions of nine trials each. During the first session, the set of extra maze cues, as well as the location of platform, was different from that on the previous place discrimination task. During the second session, the platform was moved to the new position, with the set of external cues remaining the same. Each session consisted of eight platform trials and one probe trial. The measures of platform and probe trials were the same as in the place discrimination task. The task was conducted during periods 3, 6, and 7 (see Fig. 1).

Visual discrimination task. The tank was surrounded by a black curtain to eliminate spatial cues, and rats were required to find the platform based on its visible high-contrast extension sitting $0.5 \mathrm{~cm}$ above the surface of the water. The platform was moved to different quadrants of the pool and at different distances from the walls for every trial. The start position was changed randomly for each trial. The measures of performance were the same as those for the platform trials in the place discrimination task. The test (two sessions, six trials each) was conducted at the conclusion of water maze training.

\section{Spontaneous alternation in Y-maze}

Each arm of the Y maze was $55 \times 15 \mathrm{~cm}$. The rat was placed in one arm and allowed to move freely through the maze for a $5 \mathrm{~min}$ test session. The sequence of arm entries was recorded. An alternation was defined as the number of triads containing entries into all three arms divided by the maximum possible alternations (Stone et al., 1992). After each trial, the maze was cleaned with a dilute alcohol solution and dried with a paper towel.

\section{Data analysis}

The effect of ovariectomy during periods $1-4$ was analyzed as a betweensubject main effect, with ANOVA conducted for each period separately. The effects of ovariectomy and estrogen treatments during periods 5-7 were analyzed by ANOVA with a main effect of groups (Sham, OVX and $\mathrm{OVX}_{2}$ ) and were followed by post hoc tests. The variables of performance in different sessions or delays, or after different doses of SCOP treatment, were used as repeated measures in a mixed-design ANOVA. The performance in consecutive testing periods was analyzed in a repeated-measures omnibus ANOVA. Post hoc Newman-Keuls tests were applied to significant effects and interactions to estimate the differences in the focused set of means. Some differences in the levels of degrees of freedom reported in ANOVAs reflected the data withdrawal of rats excluded from the study because of temporal or permanent health problems (fur appearance, food consumption, cataracts, etc.). When ANOVA yielded a significant effect for a set of variables (for example, all variables of platform trials), $F$ and $p$ values are presented as a range from minimum to maximum $\left(F_{(\mathrm{df})}=\right.$ minimum - maximum, minimum $<p<$ 
Figure 3. The dynamics of body weight in control (sham surgery, open squares) and experimental [ovariectomized $\mathrm{OVX}_{1}$ ( gray circles) and $\mathrm{OVX}_{2}$ (open triangles) ] groups of Fischer-344 rats during periods 1-7 of behavioral testing. A decrease in body weight during every period was attributable to food deprivation during testing in the DNMP task. Note that OVX rats gained weight compared with Sham rats, and this effect of ovariectomy was abolished quickly by both types of estrogen treatment (injections and implants).

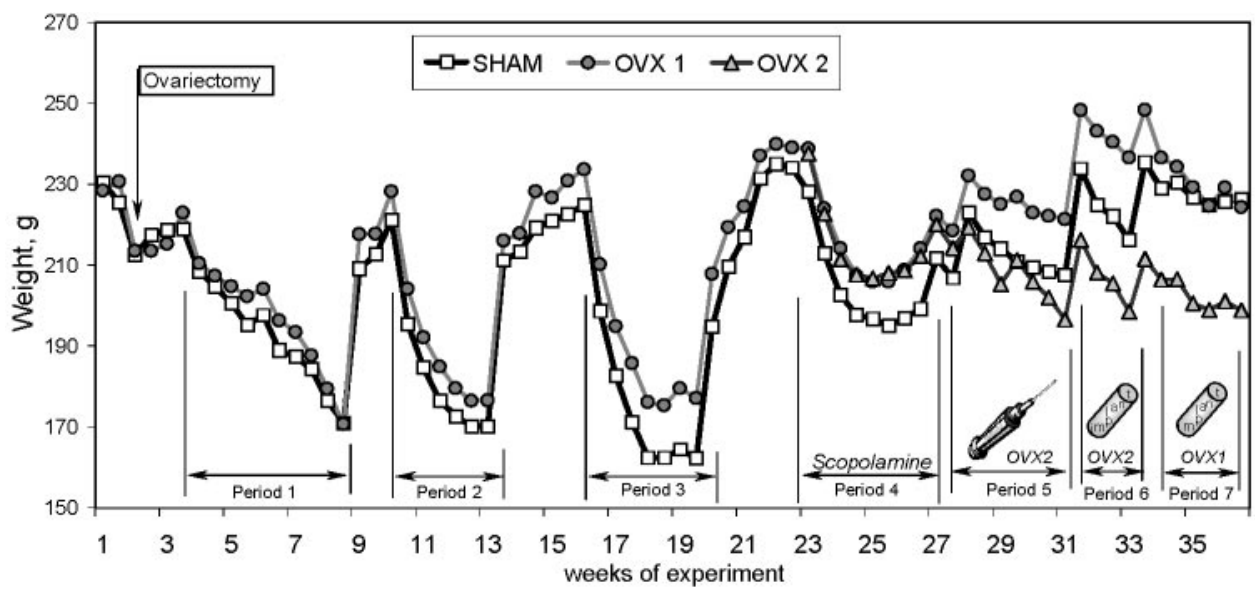

maximum). Body weight was used as a covariance to adjust the significance of between-group differences in speed of swimming. Because the effect of covariance may be overestimated if the group means for the measure used as a covariance are significantly different, additional analysis was performed using Pearson's correlation coefficient. The correlation between body weight and speed of swimming was assessed separately in Sham and OVX rats to avoid the potential confounding effect of between-group differences in body weight. All statistical analyses were conducted using Statistica 8.0 (StatSoft, Tulsa, OK).

\section{RESULTS}

\section{Changes in body weight in the course of estrogen withdrawal and replacement}

Beginning at 10 weeks after surgery, OVX rats were significantly heavier than age-matched control rats $\left(F_{(1,27)}=4.12 ; p<0.05\right)$ (Fig. 3). This difference was observed during both food deprivation periods and food ad libitum periods. The estrogen injections (group $\mathrm{OVX}_{2}$, period 5) quickly abolished the effect of ovariectomy on body weight. Following treatment with implants (period $6)$ further decreased body weight below the level of the Sham group $\left(F_{(1,11)}=12.32 ; p<0.01\right)$. Estrogen implants also decreased body weight when applied in the $\mathrm{OVX}_{1}$ group (period 7); therefore, $\mathrm{OVX}_{1}$ rats were no longer different from Sham rats $(p>0.8)$. Therefore, the gain in body weight in OVX rats was reversed easily by both modes of estrogen treatment, injections and implants, indicating that the mechanisms of regulating of body weight by estrogen remained spared with aging.

\section{Changes in estrous cycle and in plasma estrogen levels in the course of estrogen withdrawal and replacement}

At the beginning of the experiment, 13-month-old female rats showed irregular cyclicity with a high variability of the cycle duration (range, 3-10 d). However, a majority of cycles $(79 \%)$ contained a proestrus stage. In Sham rats, the number of cycles with proestrus substantially decreased at the age of 15 months $(36 \%)$ and was not detectable at the age of 18-19 months. The estrus cycles of 20- to 22-month-old Sham rats contained the long periods of diestrus ( $8-12 \mathrm{~d}$ ), infrequently followed by the short estrous stages.

Ovariectomy in 13-month-old females resulted in cessation of estrus cycle, and the vaginal smears of OVX rats were characterized by a predominance of leukocytes ( $88 \%$ of slides). Estrogen treatment (injections and/or implants) administered 7-8 months after ovariectomy resulted in vaginal cytology with a prevalence of cornified cells ( $90 \%$ of slides).

Because 13-month-old females at the beginning of the experi-

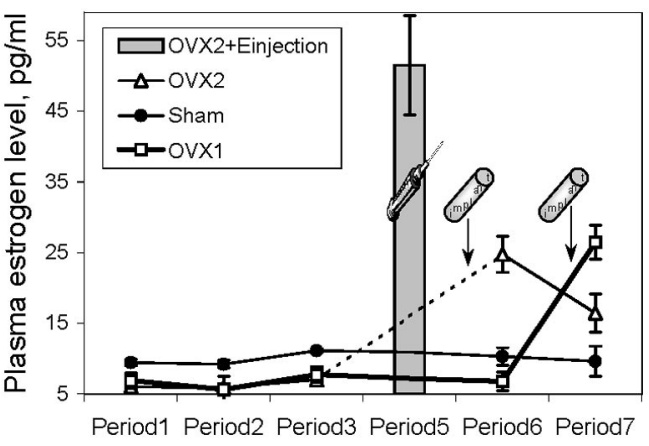

Figure 4. Plasma level of estrogen in Sham (diestrus) and OVX rats during behavioral testing (periods 1-7). The gray bar indicates the level of estrogen $1 \mathrm{hr}$ after injection of $10 \mu \mathrm{g}$ of $17 \beta$-estradiol (OVX 2 group, open triangles). The plasma estrogen concentration tested $5 \mathrm{~d}$ after implantation of pellets with $17 \beta$-estradiol was similar in both $\mathrm{OVX}_{1}$ (period 6 , open squares) and $\mathrm{OVX}_{2}$ (period 7) groups. The level of estrogen slightly decreased when tested 1 month after implantation $\left(\mathrm{OVX}_{1}\right.$ group, period 7). Filled circles, Sham.

ment already showed irregular cyclicity, and because the probability of proestrus stage decreased further with more advanced age, the plasma was collected only during diestrus. The estrogen plasma level in the Sham group was $\sim 10 \pm 1 \mathrm{pg} / \mathrm{ml}$ during diestrus and did not change significantly across all ages tested (Fig. 4). Ovariectomized rats showed a very low level of estrogen, close to the minimum detectable by the assay used. Both types of estrogen treatments, injections and implants (Fig. 4), created estrogen concentration in plasma similar to physiological levels during proestrus (Feder, 1981; Gans et al., 1995; Matsuda et al., 2002).

\section{DNMP task}

Ovariectomy increases the rate of aging-associated decline in working memory

Ovariectomy did not affect the rate of acquisition in the DNMP task. The number of sessions it took to reach the criteria of $85 \%$ of correct arm choices in 2 consecutive days was $11.5 \pm 1.6$ and $11.7 \pm 1.1$ for the Sham and OVX groups, respectively. The percentage of correct alternations of the arms increased significantly during the first $6 \mathrm{~d}$ of training $\left(F_{(5,140)}=8.12 ; p<0.0001\right)$ and was not different between groups $\left(F_{(1,28)}=1.57 ; p<0.22\right)$. The acquisition of correct choices in the stem of the T-maze (reference memory) was also similar in Sham and OVX rats $\left(F_{(1,28)}=0.60 ; p<0.44\right)$. 


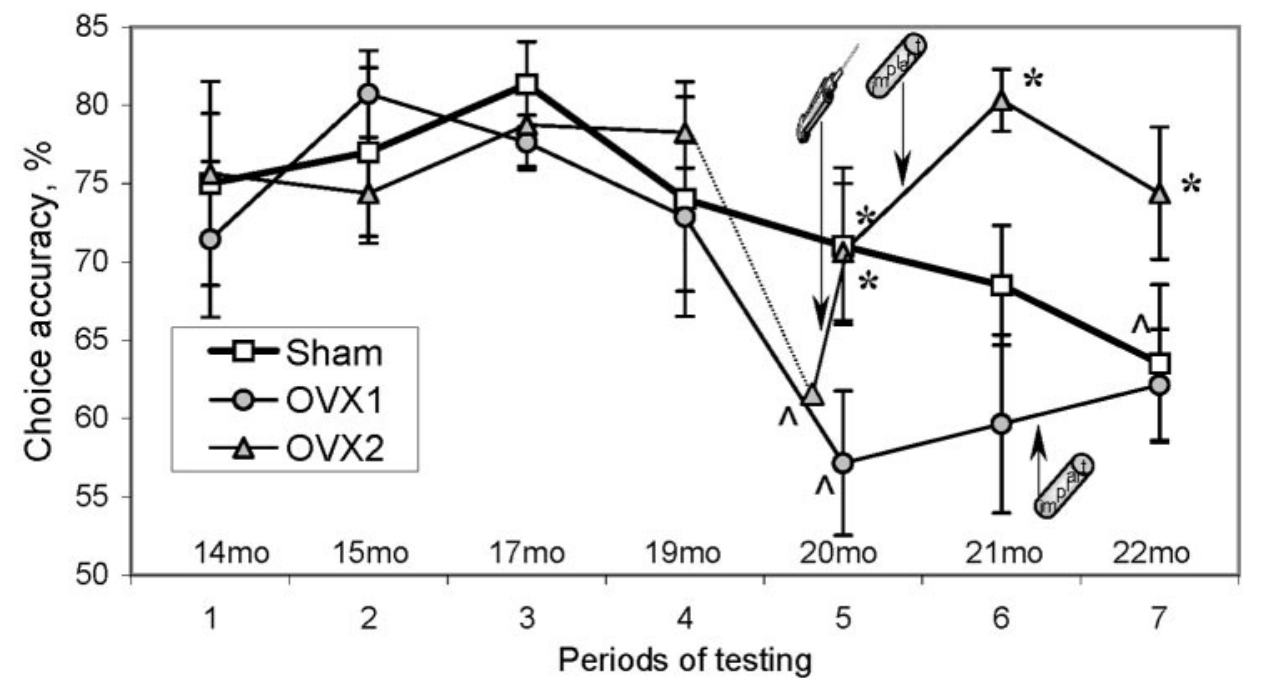

Figure 5. Aging-related changes in working memory tested in the DNMP task. Each point represents the average percentage of correct arm choices per group. Only sessions with regular, short-delay trials and without SCOP treatment are shown. The dashed line for the $\mathrm{OVX}_{2}$ group (open triangles) indicates the age-related decline before the estrogen treatment. The caret indicates the onset of the age-related decline in the appropriate group as a result of a post hoc test $(p<0.05)$. The asterisk indicates a significant difference from the $\mathrm{OVX}_{1}$ group (open circles) during the period (simple main-effect ANOVA; $p<$ 0.05). Open squares, Sham.
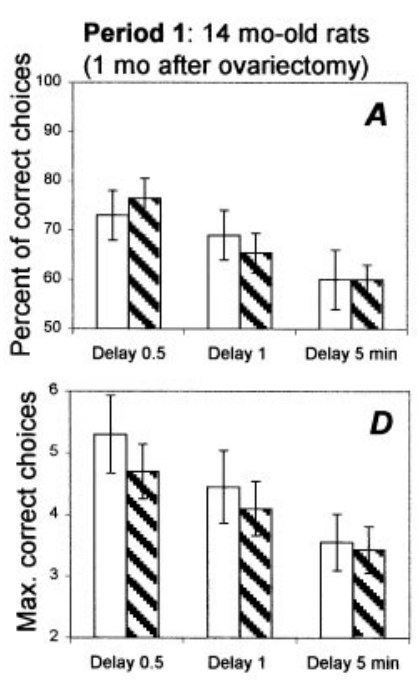

D

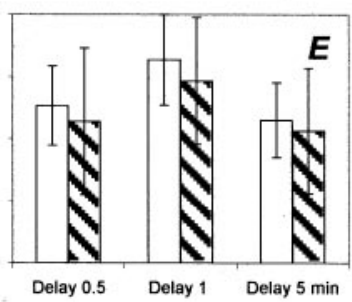

Period 2: 15 mo-old rats (2 mo after ovariectomy)

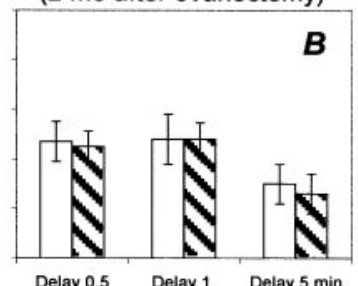

Delay 0.5 Delay 1 Delay $5 \mathrm{~min}$

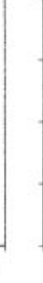

Period 3: 17 mo-old rats (4 mo after ovariectomy)
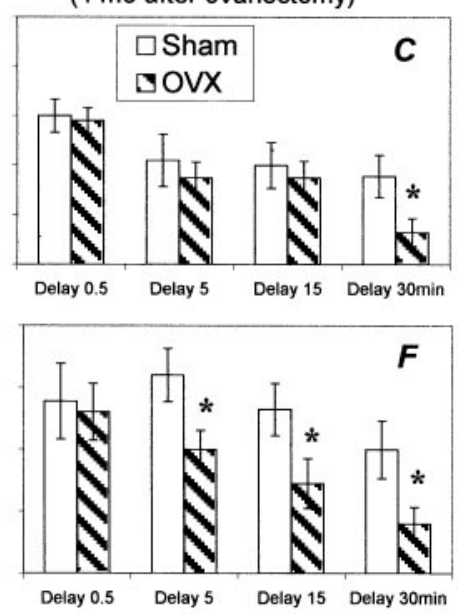

Figure 6. Delay sensitivity of working memory in Sham (open bars) and OVX (shaded bars) rats. $A-C$ represent the average percentage of correct arm choices in trials with different delays. $D-F$ represent the maximum of consecutive correct choices. Asterisks indicate significant effects of ovariectomy as a result of a simple main-effect ANOVA $(p<0.05)$.
The accuracy of correct choices in the stem of the T-maze as a measure of reference memory remained very high in the course of aging (range, 90-100\%; effect of testing periods; $p>0.6$ ) and was not different among Sham, OVX, and estrogen-treated rats ( $p$ values $>0.7)$. There were no group differences in retention of correct stem choices between consecutive periods of testing ( $p$ values $>0.3)$.

The number of correct arm choices as a measure of working memory decreased with aging. The omnibus ANOVA of choice accuracy in short-delay trials (intertrial interval, $30 \mathrm{sec}$ ) revealed a significant effect of period of testing $\left(F_{(6,102)}=4.35 ; p<0.001\right)$ and group $\left(F_{(2,17)}=6.10 ; p<0.01\right)$. However, there were no significant differences between OVX and Sham rats until 7 months after ovariectomy (group $\times$ period interaction: $F_{(12,102)}=$ 1.98; $p<0.01)$. The choice accuracy of Sham rats gradually declined after 19 months of age followed by a significant decrease at 22 months (post hoc test; $p<0.02$ ) (Fig. 5). The OVX rats showed a significant age-related deterioration in working memory at 20 months of age (post hoc test; $p<0.01$ ), i.e., 2 months earlier than Sham rats (Fig. 5).

When the demands on working memory were increased by the introduction of longer intertrial delays (1-30 min), ovariectomized rats showed a deficit in performance 4 months after ovariectomy, much earlier than that in regular, short-delay trials (Fig.
6). The introduction of 1 and 5 min delays 1 month after surgery (period 1) decreased the accuracy of arm choices (effect of delay: $\left.F_{(2,56)}=19.48 ; p<0.0001\right)$ in both Sham and OVX groups (Fig. $6 A$ ). Importantly, the accuracy of choice after the 5 min delay was close to a chance level, yielding no baseline for using longer delays. Repeated testing decreased the sensitivity of performance to delays (periods 1 and 2) (effect of retesting: $F_{(1,28)}=5.75 ; p<$ 0.03 ), which allowed the inclusion of 15 and 30 min delays during period 3 . The most challenging $30 \mathrm{~min}$ delay resulted in significant differences between OVX and Sham groups (simple main effect: $F_{(1,27)}=4.83 ; p<0.05$ ) (Fig. $6 C$ ). Even more robust between-groups differentiation was revealed in the variable of maximum consecutive correct choices (Fig. 6F). OVX rats showed significantly lower values of maximum consecutive correct choices in 5, 15, and 30 min delay trials (simple main effect; $p$ values $<0.05)$. It is important to note that the between-group differences in this variable were revealed not only in the longestdelay trials $(30 \mathrm{~min}$ ), where the total number of correct choices was significantly lower in OVX rats, but also in trials where no deficit occurred in the number of correct choices.

The data showed that the onset of ovariectomy-induced impairment in working memory was first revealed in the trials with long delays and high demands on working memory (4 months after ovariectomy, or period 3 ) and was followed by an additional 


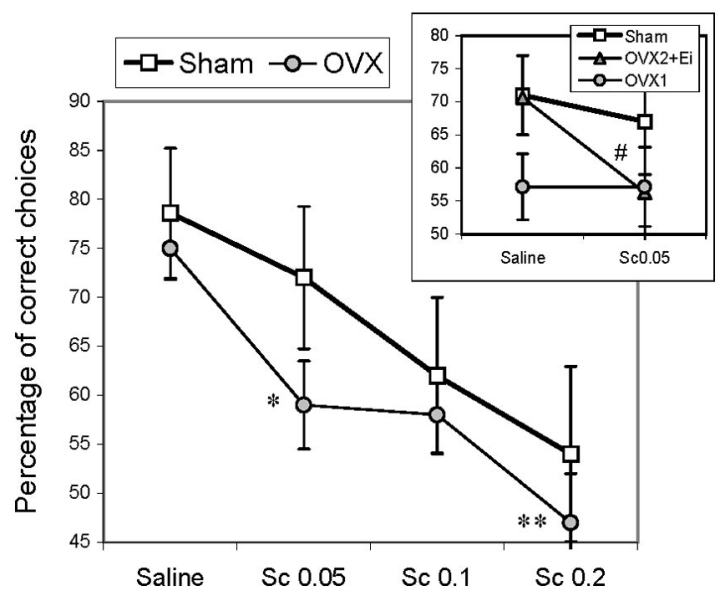

Figure 7. Effect of systemic SCOP $(S c)$ administration on the accuracy of correct arm choices in OVX (gray circles) and Sham (open squares) groups (19-month-old rats, 6 months after ovariectomy). The inset shows the effect of the lowest dose of SCOP tested 1 month later. Note that OVX rats that received estrogen treatment (group $\mathrm{OVX}_{2}$ ) were still more sensitive to SCOP than Sham rats. Single and double asterisks indicate a significant SCOP-induced decline as a result of a post hoc test applied to the significant effect of dose in the OVX group ( $p<0.01$ or 0.001 , respectively). A decline in the performance of Sham rats did not reach significance $(p>0.07)$. The pound sign indicates a significant effect of SCOP as a result of a one-way, repeated-measures ANOVA $(p<0.05)$.

decline in regular, short-delay trials with lower demand on working memory (7 months after ovariectomy, or period 5).

\section{Ovariectomy increases cognitive sensitivity to SCOP in aging rats}

To estimate the effect of compromised cholinergic functioning on T-maze alternation, both ovariectomized and control rats were injected with SCOP. Different doses of SCOP (0.05, 0.1, and 0.2 $\mathrm{mg} / \mathrm{kg}$ ) were administered systemically during period 4 of behavioral testing, when the performance of OVX rats was not different from Sham rats in regular, short-delay trials. Ovariectomized 19-month-old rats were more sensitive to the effect of SCOP than age-matched control rats. In contrast to Sham rats, OVX rats already had shown a significant decline in choice accuracy after the lowest dose of SCOP (effect of dose: $F_{(3,42)}=9.39 ; p<$ 0.0001) (Fig. 7). Sham rats decreased their choice accuracy after an increasing dose of SCOP, but this decline did not reach significance $(p>0.07)$. Moreover, the decline in choice accuracy induced by SCOP treatment remained in OVX rats for at least $3 \mathrm{~d}$, resulting in significant differences between OVX and Sham rats in sessions without SCOP injections $\left(F_{(1,18)}=5.29 ; p<0.05\right)$.

However, when treatment with estrogen improved the choice accuracy in OVX rats (period 5), the lowest dose of SCOP abolished the effect of estrogen (Fig. 7). SCOP was administered after the second and fourth sets of estrogen injections. However, in both cases the estrogen-injected $\mathrm{OVX}_{2}$ rats significantly decreased the accuracy of choices $\left(F_{(1,7)}=6.92 ; p<0.05\right)$ and were not different from $\mathrm{OVX}_{1}$ rats, with a performance close to a chance level.

\section{Efficacy of different modes of estrogen treatment in ameliorating the cognitive deficit}

After OVX rats showed a significant age-related decline in working memory in regular, short-delay probe trials (Fig. 5, period 5), one-half of ovariectomized rats $\left(\mathrm{OVX}_{2}\right)$ received subcutaneous injections of $17 \beta$-estradiol ( $10 \mu \mathrm{g}$ dissolved in $0.1 \mathrm{ml}$ of sesame
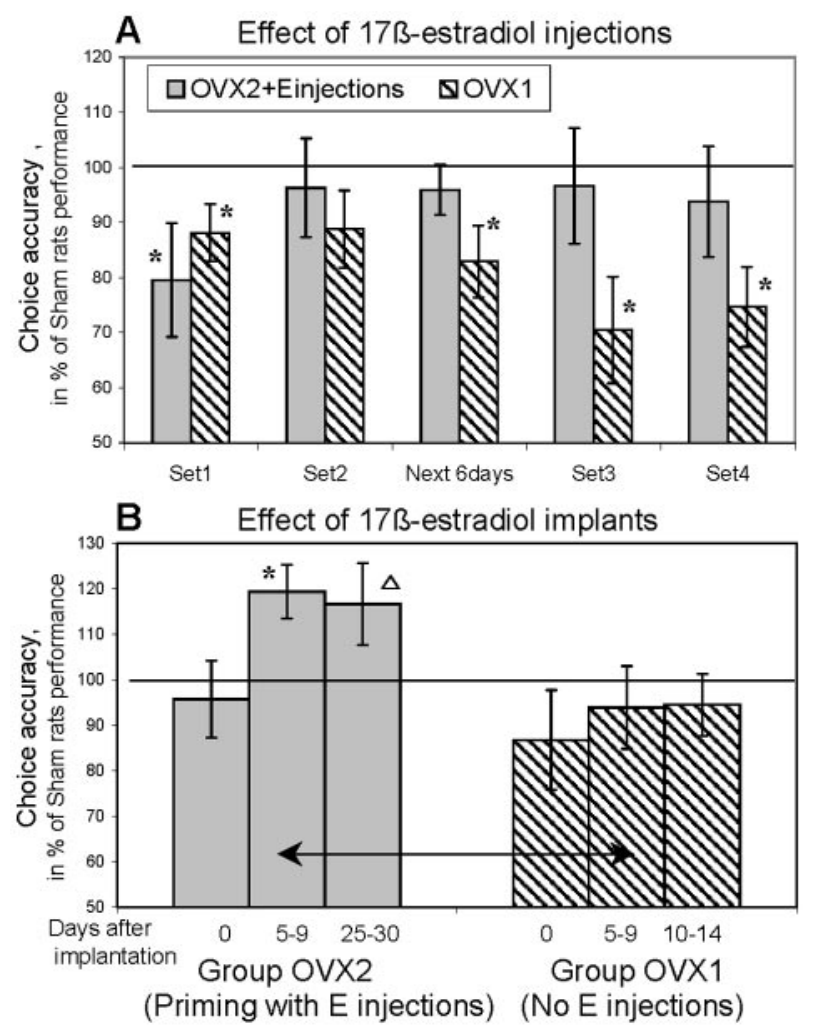

Figure 8. Effect of $17 \beta$-estradiol injections $(A)$ and implants $(B)$ on ovariectomy-induced deterioration in working memory. The accuracy of correct arm choices in control (Sham) rats is shown as $100 \%$. A, Ovariectomized rats that received repeated injections of estrogen (group $O V X 2+$ Einjections, gray bars) improved their performance to a level similar to that of control rats. The performance of ovariectomized rats that did not receive estrogen replacement $\left(\mathrm{OVX}_{1}\right.$, striped bars $)$ deteriorating further during the testing (period 5). The asterisks indicate a significant difference from control rats $(p<0.05)$. $B$, The treatment with estrogen implants was more effective in improving the performance of ovariectomized rats if treatment was preceded by estrogen injections (group $\mathrm{OVX}_{2}$, gray bars). The asterisk shows a significant increase in choice accuracy as an effect of estrogen implants; the arrow indicates a significant difference between $\mathrm{OVX}_{1}$ (striped bars) and $\mathrm{OVX}_{2}$ groups in choice accuracy between 5 and $9 \mathrm{~d}$ after implantation $(p<0.05)$. The triangle indicates the higher performance of the $\mathrm{OVX}_{2}$ group compared with the $\mathrm{OVX}_{1}$ after a longer period of estrogen treatment; however, because the exact duration of the treatment was different in the $\mathrm{OVX}_{1}$ and $\mathrm{OVX}_{2}$ groups, the difference was not statistically determined.

oil), whereas the rest of the rats $\left(\mathrm{OVX}_{1}\right)$ received subcutaneous injections of vehicle (sesame oil). $17 \beta$-Estradiol injections for 3 consecutive days constitute one set of treatment (i.e., set 1 , set 2 , etc.) (Fig. 2). The averages of choice accuracy on days 2 and 3 (after injections) are shown in Figure $8 A$. There was no effect of estrogen on choice accuracy during the first set of injections. The ovariectomized rats that received a first set of estrogen injections $\left(\mathrm{OVX}_{2}+\mathrm{E}_{\mathrm{i}}\right)$ were less accurate than control rats and were not different from other ovariectomized rats without injections (effect of group: $\left.F_{(2,17)}=4.33 ; p<0.03\right)$. The second set of estrogen injections led to a mild improvement that was followed by more robust changes during the $6 \mathrm{~d}$ without injections. During this period, the estrogen-injected rats $\left(\mathrm{OVX}_{2}+\mathrm{E}_{\mathrm{i}}\right)$ were not different from Sham rats, whereas the choice accuracy of $\mathrm{OVX}_{1}$ rats without injection was significantly lower compared with Sham rats (effect of group: $F_{(2,17)}=4.69 ; p<0.03$ ). The protective effect of estrogen injections developed gradually and was most robust after 

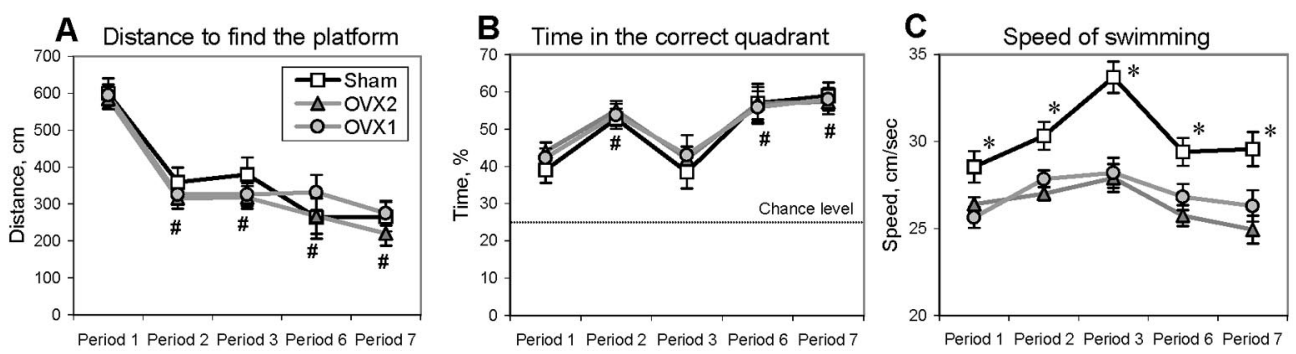

Figure 9. Performance of Sham and ovariectomized (groups $\mathrm{OVX}_{1}$ and $\left.\mathrm{OVX}_{2}\right)$ rats in platform $(A)$ and probe $(B)$ trials of the place discrimination task. The dotted line in $B$ indicates chance level. The average speed of swimming during platform trials is shown in $C$. For treatments of $\mathrm{OVX}_{1}$ (gray circles) and $\mathrm{OVX}_{2}$ (gray triangles) groups, see Figure 1. Pound signs indicate a significant improvement in place discrimination attributable to retesting

compared with period 1 ( post hoc test applied to a significant effect of period; $p<0.05$ ). Asterisks indicate a significant difference between control and ovariectomized rats $\left(\mathrm{OVX}_{1}\right.$ and $\left.\mathrm{OVX}_{2}\right)$ as a result of a post hoc test applied to a significant effect of group $(p<0.05)$. Open squares, Sham.

the third $\left(F_{(2,17)}=3.67 ; p<0.05\right)$ and fourth $\left(F_{(2,17)}=5.73 ; p<\right.$ $0.01)$ set of injections. In both cases, the significant effect of group was revealed because of the differences between Sham and OVX rats without estrogen injections.

During period 6 , the $\mathrm{OVX}_{2}$ rats, which previously received injections of estrogen, were implanted with pellets containing $17 \beta$-estradiol $\left(\mathrm{OVX}_{2}+\mathrm{E}_{\mathrm{m}}\right)$ (Fig. 1). Five days later, testing showed that rats with estrogen implants improved their performance compared with that before implantation, during period 5 (simple main effect: $F_{(1,7)}=15.75 ; p<0.01$ ). Moreover, the $\mathrm{OVX}_{2}+\mathrm{E}_{\mathrm{m}}$ group performed better than the two other groups $\left(F_{(2,17)}=6.75 ; p<0.01\right)$ (Fig. $\left.8 B\right)$. The improved choice accuracy was stable and did not change significantly when rats were tested 1 month later (period 7).

During period 7 , the remainder of the ovariectomized rats received the estrogen implants (group $\mathrm{OVX}_{1}+\mathrm{E}_{\mathrm{m}}$ ) (Fig. 1). Testing, which started $5 \mathrm{~d}$ after implantation, showed that, in contrast to the $\mathrm{OVX}_{2}$ group that received both types of estrogen treatment, the $\mathrm{OVX}_{1}$ group did not improve choice accuracy after implantation of estrogen pellets $(p>0.8)$ (Fig. $8 B$ ). Furthermore, the $\mathrm{OVX}_{1}+\mathrm{E}_{\mathrm{m}}$ rats were significantly less accurate than $\mathrm{OVX}_{2}+\mathrm{E}_{\mathrm{m}}$ rats after the same short-term period of implantation $\left(5-9\right.$ d) (effect of group: $\left.F_{(1,13)}=12.03 ; p<0.005\right)$ (Fig. $8 B$ ). Two weeks after implantation, the $\mathrm{OVX}_{1}+\mathrm{E}_{\mathrm{m}}$ group slightly improved their level of performance so that they were not different from the Sham group. However, considering that at the age of 22 months Sham rats already have shown a significant age-related decline (Fig. 5), the absence of difference between Sham and OVX $\mathrm{X}_{1}+\mathrm{E}_{\mathrm{m}}$ rats could be attributable to the decline in control rats rather than to the effects of estrogen replacement.

\section{Spontaneous alternation in the Y-maze task}

No differences were observed between Sham and OVX groups, indicating that the ovariectomized rats showed deficits in working memory compared with control rats only when higher demands on working memory were imposed, such as in the DNMP task.

\section{Place discrimination in the water maze}

No differences in spatial reference memory were observed between control and OVX rats up to 9 months after surgery (Fig. $9 A, B)$. The only ovariectomy-induced difference in place discrimination performance was observed for the variable of speed of swimming (Fig. 9C). Starting 1 month after surgery, OVX rats swam significantly slower than Sham rats $\left(F_{(1,28)}=4.69-33.32\right.$ for periods $1-7 ; 0.001<p<0.05)$. Estrogen replacement did not reduce existing differences in swim speed, although it brought back the body weight to the level of Sham rats.

In the range of ages tested (13-22 months), no effect of aging was revealed. Moreover, repeated testing improved the performance of old rats in both platform (Fig. 9A) and probe (Fig. 9B) trials compared with middle-aged naive rats (effect of period for different variables: $F_{(4,68)}=12.34-45.98 ; p$ values $\left.<0.01\right)$.

Although the place discrimination task is one of the most sensitive tasks to detect the effect of aging on reference memory (Gage et al., 1984; Fischer et al., 1992; Markowska et al., 1994; Lindner, 1997; Foster et al., 2001), repeated testing of the same animal at different time points during aging ameliorates this aging-related deficit (Beatty et al., 1985; Algeri et al., 1991; Dellu et al., 1997; Markowska, 1999a; Markowska and Savonenko, 2002). Considering that in the present study rats were tested repeatedly, the absence of an aging-related decline in place discrimination suggests that the protective effect of experience was sufficient enough to reduce the effect of aging in both Sham and ovariectomized rats.

\section{Repeated acquisition in the water maze}

The interaction of the effects of aging and repeated testing in the repeated acquisition task was more complex than in the place discrimination task. At 17 months of age, the rats were tested in this task for the first time (Fig. 10, period 3). Sham and ovariectomized rats learned the new positions of the platform and did not differ with regard to the time spent in the correct quadrant (Fig. 10A,B).

When tested at 21 months of age, preference to the correct quadrant significantly declined in both Sham and $\mathrm{OVX}_{1}$ rats (session 1) (effect of period: $F_{(1,16)}=9.32 ; p<0.01$ ) (Fig. 10A). Importantly, the memory of old rats for the new platform location was not accurate, because preference was distributed between two quadrants (Fig. 10 $\mathrm{A}$, period 6). Importantly, the second quadrant with a high preference was one of the adjacent quadrants but not the one with the old platform location. This indicates that poor memory for the platform location after the first session of the repeated acquisition task was not a result of interference with memories for the old platform location but, instead, of difficulties in learning the new position of the platform.

In the first session the change in the platform location was accompanied by the alteration in spatial cues. Notably, because only some of the spatial cues were changed, whereas others such as the geometry of the room and the location of light sources were the same, the rat's navigation could have been affected by the interference between the new and the old set of spatial cues. This additional complexity of the first session, when learning of the new platform location required the complex differentiation between interfering sets of spatial cues, was the most challenging stage of learning for 21-month-old rats.

Estrogen replacement in the $\mathrm{OVX}_{2}$ rats that received both types of treatment (injection and implants, period 6) increased the preference of the correct quadrant at the end of the first session (effect of group: $F_{(2,32)}=77.52 ; p<0.0001$ ) (Fig. 10 A). 
Figure 10. The preference for the quadrants with and without the platform tested after the first $(A)$ and second $(B)$ session in the repeated acquisition task. Each bar indicates the time spent in the quadrant with the most recent platform location (mean $\pm \mathrm{SEM}$ ). The time spent in the quadrant with a previous platform location is indicated as a marker (mean). Thick lines indicate a range of preferences for two other nonplatform quadrants. The previous correct quadrant for session 1 was a quadrant with a platform location used in the place discrimination task. See Figure 1 for treatments for the $\mathrm{OVX}_{1}$ and $\mathrm{OVX}_{2}$ groups. Significant between-groups differences in the preference for the correct quadrant were revealed only in the first session, in which a change in the platform location coincided with partial changes in spatial cues. Asterisks indicate a significant difference from Sham rats as a result of a post hoc test applied to a significant effect of group $(p<0.05)$.

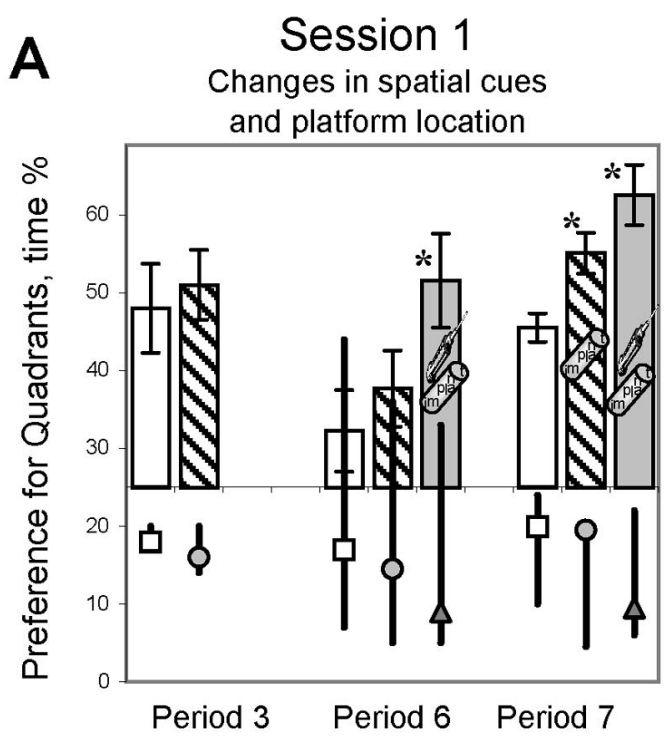

However, 21-month-old $\mathrm{OVX}_{2}+\mathrm{E}_{\mathrm{i}}+\mathrm{E}_{\mathrm{m}}$ rats, which had been equally successful in the preference of the correct quadrant as 17-month-old rats, showed as high a preference of one of the adjacent quadrants as was observed in 21-month-old Sham and $\mathrm{OVX}_{1}$ rats. This indicates that the estrogen treatment ameliorated the aging-related decline but did not change markedly the strategy used by old rats to cope with a poor memory of the new platform location.

During the second session of period 6 (Fig. 10B), when a set of spatial cues remained the same and only the platform location was changed, Sham and $\mathrm{OVX}_{1}$ rats learned the new platform location more successfully than during the first session and were no longer different from estrogen-treated $\mathrm{OVX}_{2}+\mathrm{E}_{\mathrm{i}}+\mathrm{E}_{\mathrm{m}}$ rats (session $\times$ group interaction: $\left.F_{(4,32)}=3.84 ; p<0.02\right)$. Compared with period 3, sham and ovariectomized rats improved their performance (session 2) (effect of period: $F_{(1,16)}=12.42 ; p<0.01$ ) because of the effect of retesting and/or because of a different strategy than that used by 17 -month-old naive rats (see different ranges of time spent in nonplatform quadrants in Fig. 10B, periods 3-6).

When tested 1 month later (period 7), all three groups improved performance during the first session (effect of period: $F_{(1,16)}=21.39 ; p<0.001$ ) (Fig. 10A). With additional training, 22-month-old Sham rats were able to learn the new position of the platform at a level similar to 17-month-old rats. These data are consistent with our previous results, which showed a protective effect of training against an aging-related decline in the repeated acquisition task in Fischer male rats (Markowska and Savonenko, 2002). The $\mathrm{OVX}_{2}$ rats that received both types of estrogen treatment outperformed Sham rats (effect of group: $F_{(2,16)}=4.88$; $p<0.03)$. The $\mathrm{OVX}_{1}$ rats that received estrogen implants without priming with estrogen injections showed a significantly higher preference for the correct quadrant than Sham rats ( post hoc; $p<$ $0.05)$, but they were still less accurate than the $\mathrm{OVX}_{2}$ group $(p>$ 0.1 ). Similar to the previous periods of testing, there were no differences during the second session (session $\times$ group interaction: $\left.F_{(4,32)}=2.86 ; p<0.05\right)$. Surprisingly, the preference for the correct quadrant during the second session was not improved as in period 6. The high preference for the correct quadrant during the first session (Fig. 10 $\mathrm{A}$, period 7) could have proactive effects, impairing learning on the second session. However, this view is difficult to credit, because in all groups of rats the time spent in the previous correct quadrant during the second session was at the chance level (Fig. 10B).

An alternative explanation of the performance differences between periods 6 and 7 during the second session is that the strategy applied by the rats in period 7 was different and presumably less effective when the platform was changed repeatedly. During period 6, the high preference for the correct quadrant (group $\mathrm{OVX}_{2}+\mathrm{E}_{\mathrm{i}}+\mathrm{E}_{\mathrm{m}}$ in session 1 and all groups in session 2) coincided with a low visiting (below the chance level) of the two other quadrants (the previous correct quadrant was one of those quadrants). During period 7, only one of the nonplatform quadrants was rejected, and two others (including the previous correct quadrant) were visited at the chance level. This switch in the rejection of the previous correct quadrant resulted in a strategy that would be less and less effective with consecutive changes in the platform location. As was shown in male rats, an ability to reject the previous platform location significantly declines between 18 and 24 months of age (Markowska and Savonenko, 2002). The difference in the strategies observed in females between period 6 (21 months of age) and period 7 (22 months of age) is likely also to be age-related.

Importantly, 22-month-old $\mathrm{OVX}_{2}$ rats that previously received both types of estrogen treatment were able to reject the previous platform location during the first session. However, these rats switched to the strategy inherent to age-matched controls when the platform location was changed again (session 2). These data suggest that estrogen treatment may partially ameliorate the aging-related changes in strategy rather than cause the differences in strategies between old Sham and estrogen-treated OVX rats (Fig. 10 $A$, period 7).

There were no between-group differences in latency and distance in the platform trials in any of the testing periods. All groups shortened distance and latency to find the platform during every period of testing (effect of trials; $p$ values $<0.01$ ). The pattern of differences in swim speed was similar to that in the place discrimination task.

Compared with the DNMP task, the aging-related decline in the repeated acquisition task was less robust and was ameliorated by additional training. A relatively lower demand on working memory in the repeated acquisition task, in which the information 
changes between sets of trials but not in each trial as in the DNMP task, could contribute to this difference. Importantly, unlike the DNMP task, the age-related deficit in the repeated acquisition task was not aggravated by ovariectomy. In this case, the chronic estrogen treatment (implants) was sufficient to ameliorate the aging-related changes.

\section{Straight swim and visual discrimination in the water maze}

A decrease in swim speed in the straight alley was detected in OVX rats 4 months after surgery (period 3$)\left(F_{(1,27)}=8.56 ; p<\right.$ $0.01)$. It is noteworthy that an ovariectomy-induced alteration in swim speed was detected earlier in the place discrimination task, in which an uncertainty of the situation is higher and consequently more stressful for rats than in the straight alley. This suggests that ovariectomy in middle-aged rats affected an emotional rather than a motivational component of escaping from the water.

The other factor that could affect the speed of swimming is body weight (Markowska, 1999a; Markowska and Savonenko, 2002). However, an increase in body weight was already observed 2 months earlier (period 2) without any significant impact on the speed of swimming. When body weight was used as covariance, ANCOVA still yielded a significant effect of groups in swim speed $\left(F_{(1,26)}=5.26 ; p<0.03\right)$. Moreover, there was no significant correlation between body weight and swim speed in either Sham $(r=-0.34 ; p>0.35)$ or ovariectomized groups $(r=-0.10$; $p>0.65)$.

The estrogen treatment (injections and implants; $\mathrm{OVX}_{2}$ group; period 6) did not significantly affect the swim speed compared with the nontreated ovariectomized rats $(p>0.2)$. Furthermore, at 22 months of age (period 7), when both groups of ovariectomized rats $\left(\mathrm{OVX}_{1}\right.$ and $\left.\mathrm{OVX}_{2}\right)$ received estrogen replacement for $>1$ month, the body weight of the $\mathrm{OVX}_{1}+\mathrm{E}_{\mathrm{m}}$ and $\mathrm{OVX}_{2}+\mathrm{E}_{\mathrm{m}}$ rats was similar to or lower than the body weight of Sham rats (Fig. 3). However, the speed of swimming of estrogen-treated rats was still significantly lower than that of Sham rats (effect of group; $p<0.01$ ).

There was no significant effect of ovariectomy or estrogen treatment on performance in the visual discrimination task (distance to visible platform; effect of group; $p>0.3$ ).

\section{DISCUSSION}

This study demonstrated that the rate of age-related decline in various cognitive functions was differentially affected by estrogen withdrawal. Accelerated aging in working memory function, but not in reference memory, was the most prominent consequence of estrogen withdrawal in middle-aged females. The cognitive impairment after ovariectomy was gradual (taking several months to be detected), initially occurred in tasks that placed more demands on working memory, and then was detected progressively in the easier tasks. The efficacy of estrogen in reversing the deficit in working memory as tested in the DNMP task was increased by priming the chronic estrogen administration (implants) with repeated injections, which more closely mimicked the natural estrogen cyclicity. The age-related deficit in working memory, as tested in the repeated acquisition task, was not accelerated further by estrogen withdrawal and was more responsive to both modes of estrogen replacement (injections and implants). Finally, a challenge with the muscarinic antagonist SCOP during estrogen withdrawal revealed that the ovariectomy-induced cognitive deterioration coincided with a compromised cholinergic system.
Nonetheless, the estrogen treatment that had effectively restored working memory in old ovariectomized rats failed to block the amnestic action of SCOP.

These results support the hypothesis that the sensitivity of a particular cognitive process to estrogen withdrawal parallels its sensitivity to aging. In a longitudinal design of this experiment, where the same rats are tested repeatedly, the DNMP task was the first, from a battery of cognitive tasks, to show a detrimental effect of ovariectomy. This task was also the most sensitive to the effect of aging. The cognitive sensitivity to both aging and estrogen withdrawal may depend on a working memory component in the task, with the highest demand in the DNMP task and the lowest demand in the place discrimination task. The effects of estrogen withdrawal in aging rats seemed to mirror the effects of estrogen replacement in young animals, in which estrogen facilitates performance as working memory loads increase (Bimonte and Denenberg, 1999).

Importantly, the measure of maximum consecutive correct choices in the DNMP task was the most sensitive to ovariectomyinduced changes in cognition. In contrast to regular measures of working memory (the percentage of correct choices), this measure was significantly affected in OVX rats in a wide range of delays (5-30 $\mathrm{min})$. This indicated that the ovariectomy-induced alteration in the maximum of consecutive correct choices was not attributable to the lower level of correct choices in OVX rats. The maximum number of consecutive correct choices reflects the extent to which correct performance can be maintained over a prolonged period of time. Although the DNMP task was not designed specifically to test attention span, this variable can be interpreted as a measure of sustained attention. The assessment of attention may be conducted not only by using a particular behavioral task but also by measuring the commonality engaged in different types of procedures (Bushnell, 1998). That OVX rats failed to maintain the appropriate level of vigilance in a set of consecutive trials implied that there was a possible deficit in attention. This view is consistent with results on aged ovariectomized monkeys, which showed a significant decline in attention 2 months after ovariectomy (Tinkler and Voytko, 2000). Considering the data of this study, the deficit in attention might be an initial effect of long-term estrogen withdrawal that is followed by a deficit in working memory.

An alternative explanation of ovariectomy-induced differences in the consecutive correct choices is a possible effect of estrogen withdrawal on strategies during performance in the DNMP task. One may suggest that ovariectomy could affect the balance between place/nonplace strategies that is shown to be sensitive to estrogen manipulations (Korol and Kolo, 2002). However, in this case the measure of consecutive correct choices most likely would be affected together with the total number of correct choices. Notably, when assessed in the other spatial task (repeated acquisition in the water maze), neither ovariectomy nor estrogen replacement significantly affected strategies to solve the task. However, consistent with previous studies (Barnes et al., 1980; Kobayashi et al., 1988), we observed changes in learning strategies as the animals aged. A shift in strategies observed between different periods of testing in the repeated acquisition task was most likely attributable to the age-related inability to reject the previous platform location (Markowska and Savonenko, 2002). Estrogen treatment partially ameliorated rather than directly caused this aging-related shift in strategies between old Sham and estrogen-treated OVX rats.

At the age at which memory impairment was detected only in 
OVX rats but not in Sham rats (17-20 months), the functions of ovaries in Sham rats already had been compromised substantially by aging, as indicated by the termination of the proestrus stage. Thus, the differences in working memory between old OVX and Sham rats were not caused by the actual drop in estrogen at the time of testing but rather by consequences of estrogen withdrawal at a younger age, when ovaries were more functional. The persistence of the effect of estrogen well beyond the period of exposure has also been documented in the working memory of young rats (Daniel et al., 1997). In Sham rats with a natural aging-related decline in neuroendocrine functions, the onset of the cognitive deficit was also delayed by 3-4 months after a cessation of the proestrus stage. Considering that the surgical estrogen withdrawal resulted in a cognitive deficit 4 months after surgery, the same delayed principle of the effect of natural estrogen withdrawal has been observed in normally aging subjects.

The central cholinergic system is a good example of a system in which the interaction of the effects of the aging processes (Biegon et al., 1986; Fischer et al., 1992; Olton and Markowska, 1992; Olton et al., 1992; Markowska et al., 1995) and estrogen withdrawal (Fader et al., 1999; Gibbs, 1999; Miller et al., 1999) may affect cognition. In the present study, the ovariectomized rats with an accelerated rate of cognitive aging were more sensitive than the Sham rats to a SCOP-induced impairment in working memory. They were also impaired in the ability to restore normal cognitive functions after SCOP treatment. More importantly, the ability of the estrogen treatment to restore working memory in old rats was not paralleled by appropriate changes in sensitivity to the amnestic action of SCOP. The minimal dose of SCOP, which was sufficient to impair the performance of ovariectomized rats in the DNMP task, was still effective after the estrogen treatment. These data clearly indicate that the estrogen treatment that had been effective in restoring the cognitive abilities of old rats did not cause changes in cholinergic function to counter the effects of SCOP. Our results are consistent with a previous study (Gibbs, 2000b), showing that estrogen failed to reduce the SCOP-induced deficits in the delayed-matching-to-position task when tested in 22- to 25-month-old female rats. Because estrogen prevents the impairment in working memory after SCOP if administered in younger rats (Fader et al., 1999; Gibbs, 1999), these data clearly indicate that the mechanisms underlying the protective effect of estrogen replacement may be different when applied in young and old subjects. This view is supported by data showing an agingrelated decline in the ability of estrogen to stimulate cholinergic (Singer et al., 1998) and neurotrophin (Jezierski and Sohrabji, 2001) functions. Considering that estrogen has multipotent effects in a variety of neuromediator systems (McEwen et al., 1997), and that the aging processes can differentially affect various systems, the effects of estrogen treatment at old age are more likely to rely on mechanisms that were relatively spared during aging. Therefore, the strategy for treatment of cognitive consequences of neuroendocrine imbalance in aged females should also consider other neuromediator systems, which may have been relatively spared during aging.

The question of whether the deficit in working memory induced by long-term estrogen withdrawal can be ameliorated or reversed by estrogen treatment if applied at advanced ages has very important clinical implications. The enhancing effect of estrogen on working memory was documented repeatedly in young rats (O'Neal et al., 1996; Daniel et al., 1997; Bimonte and Denenberg, 1999). In the present study, the chronic estrogen treatment (implants) did not improve cognitive abilities in old ovariectomized rats when a cognitive deficit already had been detected in the DNMP task. These results are in accordance with clinical data showing a limited role of estrogen replacement therapy in cognitive enhancement in women already diagnosed with Alzheimer's disease (Henderson et al., 2000; Mulnard et al., 2000; Wang et al., 2000). Importantly, the same estrogen treatment was still effective in old rats when a cognitive process was relatively spared during aging and was not aggravated by ovariectomy, as was shown in the repeated acquisition task.

However, when treatment with implants was primed with estrogen injections, the working memory of old ovariectomized rats was improved significantly, indicating that cognitive sensitivity to estrogen treatment at advanced age could be restored. This also indicated that a low effectiveness of estrogen implants in old rats was not caused by an insufficient level of estrogen but, rather, by a lack of readiness of the organism to respond to such treatment. The mechanisms of priming with estrogen injections might be related to the cyclical nature of the replacement as well as to the high levels (although brief) of estrogen in the first minutes after injections. Although additional studies are needed to differentiate between these possibilities, the present data clearly indicate that the efficacy of estrogen treatment on the cognition of old rats is highly successful with a phasic but not tonic replacement paradigm. In view of the negative results of the recently halted clinical trial in which estrogen (together with progesterone) was administered daily (Fletcher and Colditz, 2002), the results of the present study strongly advocate a phasic (cyclic) administration of estrogen. This paradigm appears to be highly beneficial for cognitive improvement and may produce fewer side effects than continuous daily administration.

\section{REFERENCES}

Algeri S, Biagini L, Manfridi A, Pitsikas N (1991) Age-related ability of rats kept on a life-long hypocaloric diet in a spatial memory test. Longitudinal observations. Neurobiol Aging 12:277-282.

Barnes CA, Nadel L, Honig WK (1980) Spatial memory deficit in senescent rats. Can J Psychol 34:29-39.

Beatty WW, Bierley RA, Boyd JG (1985) Preservation of accurate spatial memory in aged rats. Neurobiol Aging 6:219-225.

Biegon A, Greenberger V, Segal M (1986) Quantitative histochemistry of brain acetylcholinesterase and learning rate in the aged rat. Neurobiol Aging 7:215-217.

Bimonte HA, Denenberg VH (1999) Estradiol facilitates performance as working memory load increases. Psychoneuroendocrinology 24:161-173.

Bushnell PJ (1998) Behavioral approaches to the assessment of attention in animals. Psychopharmacology (Berl) 138:231-259.

Daniel JM, Fader AJ, Spencer AL, Dohanich GP (1997) Estrogen enhances performance of female rats during acquisition of a radial arm maze. Horm Behav 32:217-225.

Dellu F, Mayo W, Vallee M, Le Moal M, Simon H (1997) Facilitation of cognitive performance in aged rats by past experience depends on the type of information processing involved: a combined cross-sectional and longitudinal study. Neurobiol Learn Mem 67:121-128.

Ditkoff EC, Crary WG, Cristo M, Lobo RA (1991) Estrogen improves psychological function in asymptomatic postmenopausal women. Obstet Gynecol 78:991-995.

Dohanich GP, Fader AJ, Javorsky DJ (1994) Estrogen and estrogenprogesterone treatments counteract the effect of scopolamine on reinforced T-maze alternation in female rats. Behav Neurosci 108:988-992.

Fader AJ, Hendricson AW, Dohanich GP (1998) Estrogen improves performance of reinforced T-maze alternation and prevents the amnestic effects of scopolamine administered systemically or intrahippocampally. Neurobiol Learn Mem 69:225-240.

Fader AJ, Johnson PE, Dohanich GP (1999) Estrogen improves working but not reference memory and prevents amnestic effects of scopolamine of a radial-arm maze. Pharmacol Biochem Behav 62:711-717.

Feder HH (1981) Estrous cyclicity in mammals. In: Neuroendocrinology of reproduction: physiology and behavior (Adler NT, ed), pp 179-345. New York: Plenum.

Fischer W, Chen KS, Gage FH, Bjorklund A (1992) Progressive decline in spatial learning and integrity of forebrain cholinergic neurons in rats during aging. Neurobiol Aging 13:9-23. 
Fletcher SW, Colditz GA (2002) Failure of estrogen plus progestin therapy for prevention. JAMA 288:366-368.

Foster TC, Sharrow KM, Masse JR, Norris CM, Kumar A (2001) Calcineurin links $\mathrm{Ca}^{2+}$ dysregulation with brain aging. J Neurosci 21:4066-4073.

Gage FH, Dunnett SB, Bjorklund A (1984) Spatial learning and motor deficits in aged rats. Neurobiol Aging 5:43-48.

Gans SE, Stamper JL, Butler T, McClintock MK (1995) Endocrine basis for two types of individual differences in lordosis reflex intensity. Horm Behav 29:367-391.

Gibbs RB (1998) Impairment of basal forebrain cholinergic neurons associated with aging and long-term loss of ovarian function. Exp Neurol 151:289-302.

Gibbs RB (1999) Estrogen replacement enhances acquisition of a spatial memory task and reduces deficits associated with hippocampal muscarinic receptor inhibition. Horm Behav 36:222-233.

Gibbs RB (2000a) Effects of gonadal hormone replacement on measures of basal forebrain cholinergic function. Neuroscience 101:931-938.

Gibbs RB (2000b) Long-term treatment with estrogen and progesterone enhances acquisition of a spatial memory task by ovariectomized aged rats. Neurobiol Aging 21:107-116.

Gibbs RB, Aggarwal P (1998) Estrogen and basal forebrain cholinergic neurons: implications for brain aging and Alzheimer's disease-related cognitive decline. Horm Behav 34:98-111.

Gorzalka BB, Moe IV (1994) Adrenal role in proceptivity and receptivity induced by two modes of estradiol treatment. Physiol Behav 55:29-34.

Grady D, Herrington D, Bittner V, Blumenthal R, Davidson M, Hlatky M, Hsia J, Hulley S, Herd A, Khan S, Newby LK, Waters D, Vittinghoff E, Wenger N (2002) Cardiovascular disease outcomes during 6.8 years of hormone therapy: heart and estrogen/progestin replacement study follow-up (HERS II). JAMA 288:49-57.

Henderson VW, Paganini-Hill A, Miller BL, Elble RJ, Reyes PF, Shoupe D, McCleary CA, Klein RA, Hake AM, Farlow MR (2000) Estrogen for Alzheimer's disease in women: randomized, double-blind, placebocontrolled trial. Neurology 54:295-301.

Hepler DJ, Olton DS, Wenk GL, Coyle JT (1985) Lesions in nucleus basalis magnocellularis and medial septal area of rats produce qualitatively similar memory impairments. J Neurosci 5:866-873.

Hulley S, Furberg C, Barrett-Connor E, Cauley J, Grady D, Haskell W, Knopp R, Lowery M, Satterfield S, Schrott H, Vittinghoff E, Hunninghake D (2002) Noncardiovascular disease outcomes during 6.8 years of hormone therapy (HERS II). JAMA 288:58-66.

Jezierski MK, Sohrabji F (2001) Neurotrophin expression in the reproductively senescent forebrain is refractory to estrogen stimulation. Neurobiol Aging 22:309-319.

Kimura D (1995) Estrogen replacement therapy may protect against intellectual decline in postmenopausal women. Horm Behav 29:312-321.

Kobayashi S, Kametani H, Ugawa Y, Osanai M (1988) Age difference of response strategy in radial maze performance of Fischer-344 rats. Physiol Behav 42:277-280.

Korol DL, Kolo LL (2002) Estrogen-induced changes in place and response learning in young adult female rats. Behav Neurosci 116:411-420.

Lindner MD (1997). Reliability, distribution, and validity of age-related cognitive deficits in the Morris water maze. Neurobiol Learn Mem 68:203-220.

Luine VN, Richards ST, Wu VY, Beck KD (1998) Estradiol enhances learning and memory in a spatial memory task and effects levels of monoaminergic neurotransmitters. Horm Behav 34:149-162.

Markowska AL (1999a) Life-long diet restriction failed to retard cognitive aging in Fischer-344 rats. Neurobiol Aging 20:177-189.

Markowska AL (1999b) Sex dimorphisms in the rate of age-related decline in spatial memory: relevance to alterations in the estrous cycle. J Neurosci 19:8122-8133.

Markowska AL, Grinnell D (1999) Hormonal interaction with cognition during aging. Soc Neurosci Abstr 25:1062.

Markowska AL, Savonenko AV (2002) Protective effect of practice on cognitive decline during aging: implications for its efficacy. Neurobiol Learn Mem 78:294-320.

Markowska AL, Wenk GL, Olton DS (1990) Nucleus basalis magnocellularis and memory: differential effects of two neurotoxins. Behav Neural Biol 54:13-26.
Markowska AL, Long JM, Johnson CT, Olton DS (1993) Variableinterval probe test as a tool for repeated measurements of spatial memory in the water maze. Behav Neurosci 107:627-632.

Markowska AL, Koliatsos VE, Breckler SJ, Price DL, Olton DS (1994) Human nerve growth factor improves spatial memory in aged but not in young rats. J Neurosci 14:4815-4824.

Markowska AL, Olton DS, Givens BA (1995) Cholinergic manipulations in the medial septal area: age-related effects on working memory and hippocampal electrophysiology. J Neurosci 15:2063-2073.

Markowska AL, Mooney M, Sonntag WE (1998) Insulin-like growth factor-1 ameliorates age-related behavioral deficits. Neuroscience 87:559-569.

Matsuda Y, Hirano H, Watanabe Y (2002) Effect of estrogen on acetylcholine release in frontal cortex of female rats: involvement of serotonergic neuronal systems. Brain Res 937:58-65.

McEwen BS, Alves SE, Bulloch K, Weiland NG (1997) Ovarian steroids and the brain: implications for cognition and aging. Neurology 48:S8-S15.

Miller MM, Hyder SM, Assayag R, Panarella SR, Tousignant P, Franklin KB (1999) Estrogen modulates spontaneous alternation and the cholinergic phenotype in the basal forebrain. Neuroscience 91:1143-1153.

Mulnard RA, Cotman CW, Kawas C, van Dyck CH, Sano M, Doody R, Koss E, Pfeiffer E, Jin S, Gamst A, Grundman M, Thomas R, Thal LJ (2000) Estrogen replacement therapy for treatment of mild to moderate Alzheimer disease: a randomized controlled trial. Alzheimer's Disease Cooperative Study. JAMA 283:1007-1015.

Olton DS, Markowska AL (1992) The aging septo-hippocampal system: its role in age-related memory impairments. In: Neuropsychology of memory, Ed 2 (Squire LR, Butters N, eds), pp 378-385. New York: Guilford.

Olton DS, Givens BS, Markowska AL, Shapiro M, Golski S (1992) Mnemonic functions of the cholinergic septohippocampal system. In: Memory: organization and locus of change (Squire LR, Weinberger NM, McGaugh JL, eds). London: Oxford UP.

O'Neal MF, Means LW, Poole MC, Hamm RJ (1996) Estrogen affects performance of ovariectomized rats in a two-choice water-escape working memory task. Psychoneuroendocrinology 21:51-65.

Reilly CM, Cannady WE, Mahesh VB, Stopper VS, De Sevilla LM, Mills TM (1996) Duration of estrogen exposure prior to follicle-stimulating hormone stimulation is critical to granulosa cell growth and differentiation in rats. Biol Reprod 54:1336-1342.

Sherwin BB (1988) Estrogen and/or androgen replacement therapy and cognitive functioning in surgically menopausal women. Psychoneuroendocrinology 13:345-357.

Sherwin BB (1994) Estrogenic effects on memory in women. Ann NY Acad Sci 743:213-230.

Singer CA, McMillan PJ, Dobie DJ, Dorsa DM (1998) Effects of estrogen replacement on choline acetyltransferase and trkA mRNA expression in the basal forebrain of aged rats. Brain Res 789:343-346.

Singh M, Meyer EM, Millard WJ, Simpkins JW (1994) Ovarian steroid deprivation results in a reversible learning impairment and compromised cholinergic function in female Sprague-Dawley rats. Brain Res 644:305-312.

Stone WS, Rudd RJ, Gold PE (1992) Glucose attenuation of scopolamine- and age-induced deficits in spontaneous alternation behavior and regional brain $\left[{ }^{3} \mathrm{H}\right] 2$-deoxyglucose uptake in mice. Psychobiology 20:270-279.

Thigpen JE, Setchell KD, Ahlmark KB, Locklear J, Spahr T, Caviness GF, Goelz MF, Haseman JK, Newbold RR, Forsythe DB (1999) Phytoestrogen content of purified, open- and closed-formula laboratory animal diets. Lab Anim Sci 49:530-536.

Tinkler GP, Voytko ML (2000) Multiple cognitive domains are affected by estrogen state in middle-aged surgically menopausal monkeys. Soc Neurosci Abstr 26:1745.

Wang PN, Liao SQ, Liu RS, Liu CY, Chao HT, Lu SR, Yu HY, Wang SJ, Liu HC (2000) Effects of estrogen on cognition, mood, and cerebral blood flow in AD: a controlled study. Neurology 54:2061-2066.

Woolley CS, McEwen BS (1994) Estradiol regulates hippocampal dendritic spine density via an $N$-methyl-D-aspartate receptor-dependent mechanism. J Neurosci 14:7680-7687. 